\title{
Consolidation of statistical information of multiple objects in working memory
}

\author{
Shruti Baijal • Narayanan Srinivasan
}

Published online: 24 May 2011

(C) Psychonomic Society, Inc. 2011

\begin{abstract}
The present study investigated working memory consolidation in focused and distributed attention tasks by examining the time course of the consolidation process (Experiment 1) and its dependence on capacity-limited central resources (Experiment 2) in both tasks. In a match-to-sample design using masks at various intervals to vary consolidation rates, the participants performed either an identification task (focused attention) or a mean estimation task (distributed attention) with (Experiment 1) or without (Experiment 2) prior knowledge of what task they were to perform. We found that consolidation in the distributed attention task was more efficient and was about twice as fast as in the focused attention task. In addition, both tasks suffered interference when they had to be performed together, indicating that both types of attention rely on a common set of control processes. These findings can be attributed to differences in the resolution of object representations and in the scope of attention associated with focused and distributed attention.
\end{abstract}

Keywords Attention - Consolidation - Selection - Statistical judgment $\cdot$ Working memory

Active encoding of relevant information into working memory is susceptible to attentional constraints. In other words, there are limitations in the process of active encoding of perceptual representations into durable working memory representations, a process also termed consolidation (Chun \& Potter, 1995; Jolicœur \& Dell'Acqua, 1998; Vogel, Luck, \& Shapiro, 1998). For example, research on the attentional blink is consistent with the

S. Baijal $(\bowtie) \cdot$ N. Srinivasan

Centre of Behavioural and Cognitive Sciences,

University of Allahabad,

Allahabad U.P. 211002, India

e-mail: shrutz.b@gmail.com suggestion that consolidation in working memory is capacity limited. Similarly, our ability to select and track randomly moving objects is also limited (Cavanagh \& Alvarez, 2005). It has been suggested that attention is the capacity-limited process that restricts processing in working memory (Cowan, 1998, 2001). However, it is not yet clear how differences in the scope or the type of attention affect consolidation of information in working memory.

Previous studies on the temporal dynamics of consolidation in working memory with tasks that involved focused attention have reported that it takes about $500 \mathrm{~ms}$ to form the working memory representation of a single item (Chun \& Potter, 1995; Jolicœur \& Dell'Acqua, 1998; Ward, Duncan, \& Shapiro, 1996). This duration increases with increases in the number of consolidated items (Jolicœur \& Dell'Acqua, 1998; Vogel, Woodman, \& Luck, 2006).

Jolicœur and Dell'Acqua (1998) used a dual-task paradigm that combined a verbal working memory task with an auditory speeded-response task. The observers were first visually presented with a memory array of alphanumeric characters (Target 1 [T1]), and this was followed by a low- or high-pitched tone (Target 2 [T2]). The observers made a speeded response to T2, after which memory for $\mathrm{T} 1$ was tested. Jolicœur and Dell'Acqua found that responses to $\mathrm{T} 1$ were slow when the delay between $\mathrm{T} 1$ and $\mathrm{T} 2$ was short (350-500 ms). However, the responses became progressively faster as the delay between $\mathrm{T} 1$ and $\mathrm{T} 2$ increased, eventually reaching an asymptote. In addition, the responses to $\mathrm{T} 2$ were slower when the observers had to remember multiple items rather than a single item, and this difference was largest at short delays. These results suggest that working memory consolidation of the memory array and response selection involve similar capacity-limited central mechanisms. In other words, the interference between consolidation of $\mathrm{T} 1$ and response selection of $\mathrm{T} 2$ 
resulted in slower consolidation, thus requiring more time when more items must be remembered. However, it was unclear whether the slowing at short delays was due to both consolidation and response selection processes being affected, or to just one of them being affected.

Recently, Vogel et al. (2006) used a visual working memory paradigm that required observers to focus attention on two arrays of colored squares separated by a $900-\mathrm{ms}$ interval. The task was to report whether the arrays were the same or the color of one of the squares had changed. Consolidation of the memory array was interrupted at different time intervals using a pattern mask. The researchers found that consolidation is actually a relatively rapid process that can form about three durable visual working memory representations within $200 \mathrm{~ms}$ of visual processing. Their measure of the consolidation rate of $50 \mathrm{~ms}$ per item was derived from a single-task procedure and may have been more directly related to the time of consolidation without the influence of a second competing task. This result suggests that working memory consolidation is a relatively rapid process whose rate is comparable to the rate of attentional scanning in visual search (Treisman, 1988; Wolfe, 1994; Woodman \& Luck, 1999). This supports the idea that consolidation in working memory and attention in visual search are supported by the same process (Cowan, 2001), and the rate of consolidation may vary depending on the nature of the deployment of attention.

Earlier evidence showed that consolidation rates may vary depending on the nature of the information that is being extracted (Potter, 1976). Potter conducted a series of experiments to measure the time required for perceptual identification and memory encoding. A set of 16 photographs of complex scenes were presented in a rapid serial visual presentation (RSVP) paradigm. The presentation rates were one, three, four, six, or eight stimuli per second. In one of the experiments, the observers had to indicate whether they detected the scene that matched the word presented before the sequence. The accuracy remained high at all stimulus presentation rates. These results cannot be attributed to the detection of low-level features in the scene, but result from identification at the conceptual level. It was concluded that the visual system could rapidly extract the gist of a scene in order to form its conceptual representation. However, this pattern of results could be due to faster identification of the gist of a scene rather than to faster consolidation. Therefore, it remains unclear how much time is needed for consolidation of the gist and statistical information in a visual scene.

It has been proposed that extraction of the gist of a scene and its statistical properties involves distributed attention, putatively different from the typical construct of focused attention (Baijal \& Srinivasan, 2009; Srinivasan, Srivastava, Lohani, \& Baijal, 2009; Treisman, 2006). The process of extracting statistical information (average size or orientation) and gist appears to be quick, effortless, parallel, and early in the course of the visual processing stream (Chong, Joo, Emmanouil, \& Treisman, 2008; Chong \& Treisman, 2003, 2005a; Im \& Chong, 2009). However, many aspects of the averaging process guided by distributing attention over multiple objects are not clear.

Some evidence for differences between focused and distributed attention comes from earlier studies. Baijal, Nakatani, Srinivasan, and van Leeuwen (2009) found evidence to indicate differences in the control of access to working memory representations maintained during a focused, as compared to a distributed, attention task. The observers were presented with a display of a variable number of circles of different sizes. Later, their memory was tested for either the individual sizes (focused attention) or the average size (distributed attention) of the circles from the previous display. As expected, the working memory limitations were less severe when distributed attention was deployed. More importantly, in terms of brain activity measured using contralateral delay activity, it was found that focused attention has an adjustable scope that increases up to the limit of about four targets. On the other hand, distributed attention has a wide scope, irrespective of the number of targets (Baijal et al., 2009).

In a multiple-object-tracking task (Alvarez \& Oliva, 2008) that required focused attention to moving targets and updating of object locations in working memory, participants reported the location either of a single moving object or of the centroid (or average position) of a group of moving objects. While for a single object, the localization was better for the target than distractor, the localization performance of the centroid of multiple targets and that for the distractors was observed to be well above chance. The authors suggested that some form of attention could be paid to the distractors (other than focused attention) that did not benefit localization of an individual distractor but facilitated localization of their pooled representation. Similar results were obtained even when the target selection was made easy. The study demonstrated that even though the local details were poorly represented and identified at chance levels, the pooling of local features to obtain statistical information could be achieved, resulting in better performance (Alvarez \& Oliva, 2008).

Performance in an averaging task utilizing distributed attention is little, if at all, affected by variation in the number or density of items (Chong \& Treisman, 2005a), exposure duration or delay (Chong \& Treisman, 2003), and the difficulty of selection (Chong \& Treisman, 2005b). These results contrast with findings on the capacity limitations associated with working memory involving focused attention (Baddeley, 1997; Franconeri, Alvarez, \& 
Enns, 2007; Luck \& Vogel, 1997; Makovski \& Jiang, 2007).

One important question related to the computation of statistical properties using distributed attention is the time course of statistical computations - that is, the time taken to complete the averaging computations and store that information in working memory. Although averages (like mean size) can be estimated from a 50-ms presentation (Chong \& Treisman, 2003), there might also be contributions from late visual representations, since the studies that have investigated this question did not employ masking to disrupt further processing. While the averaging process is more accurate when attention is distributed than when it is focused, this by itself does not say anything about the temporal differences in processing associated with these different types of attention.

Given the paucity of studies of distributed attention, we investigated the rate of consolidation in working memory with focused and distributed attention. This would in turn reveal how different types of attention enable the encoding of input into working memory. Therefore, in the present study we estimated the time courses of working memory consolidation when focused and distributed attention are deployed. Moreover, we also investigated whether focused and distributed attention tasks recruited the same capacitylimited central mechanisms. In the present study, we examined these questions by comparing the rates of consolidation for both tasks (Experiment 1) and then estimating whether the two tasks competed with each other for central processing resources (Experiment 2).

\section{Experiment 1}

In Experiment 1, we sought to provide an initial estimate of the time course of visual working memory consolidation during focused attention and distributed attention tasks. We used a procedure similar to the one used by Vogel et al. (2006), although the tasks were changed according to the demands of the present study. The consolidation of visual representations in working memory was interrupted by presenting pattern masks after the disappearance of the memory array. The time interval between the presentation of the memory array and the mask was varied and chosen based on previous studies (Vogel et al., 2006). A second later, the memory was tested for both of the tasks to observe the influence of types of attention on working memory consolidation. Previous studies have shown that estimating the mean of multiple elements is a relatively rapid process as compared to processing individuated elements (Chong \& Treisman, 2003; Treisman, 2006). Therefore, we expected that focused and distributed attention tasks would be associated with different rates of working memory consol- idation, with consolidation being faster in the latter than in the former.

\section{Method}

Participants A group of 18 volunteers from the University of Allahabad with normal or corrected-to-normal vision participated in the experiment.

Stimuli and apparatus All stimuli were presented on a monitor screen viewed from a distance of $80 \mathrm{~cm}$. The stimulus display consisted of either two or four red-colored (RGB [220, 71, 105]) disks with luminance $9.4 \mathrm{~cd} / \mathrm{m}^{2}$. The circumference of any two disks was separated by at least $1.43^{\circ}$ of visual angle. The circles were placed at $2.86^{\circ}$ (distance from circumference) from the central fixation cross. The background was gray (RGB [192, 192, 192]), and its luminance was $26.6 \mathrm{~cd} / \mathrm{m}^{2}$. The diameter of the disks ranged from $0.29^{\circ}$ to $2.29^{\circ}$ with a step size of $0.19^{\circ}$, which yielded 11 sizes. These sizes were chosen pseudorandomly for the stimulus display. The stimulus display never contained a disk whose size matched the average size of the target set. The stimulus display was followed by a test display that consisted of two red disks. The size of one of the disks matched the correct answer (which will be explained below), while the other was either larger or smaller than the correct disk by $0.39^{\circ}$. The mask was generated by selecting the location of the stimulus display (roughly ring-shaped, centered at fixation) and filling it with gray and black blotches. The stimulus presentation and data collection were performed using DirectRT (Empirisoft Corp., New York, NY).

Procedure Each trial began with a central fixation cross presented for $500 \mathrm{~ms}$, followed by 33-ms presentation of a memory array of two or four disks that varied from trial to trial. Next, there was a variable-duration interstimulus interval (ISI), followed by a 500-ms-duration mask display. The stimulus onset asynchronies (SOAs) were thus 66, 133, and $250 \mathrm{~ms}$, each used in one-third of the trials. The ISI between the memory array and the test array was always $1,000 \mathrm{~ms}$, regardless of the memory-array-to-mask ISI, because we concurrently adjusted the time between the mask and the test array (see Fig. 1).

The member task (focused attention) and the mean task (distributed attention) with the memory disks were blocked (two blocks of trials for each task), and the order of the blocks was randomized across participants. During the member task, the participants indicated which of two test disks matched the size of the disks presented in the memory array. During the mean task, the participants indicated which of two test disks matched the mean size of all the disks presented in the memory array. The participants 


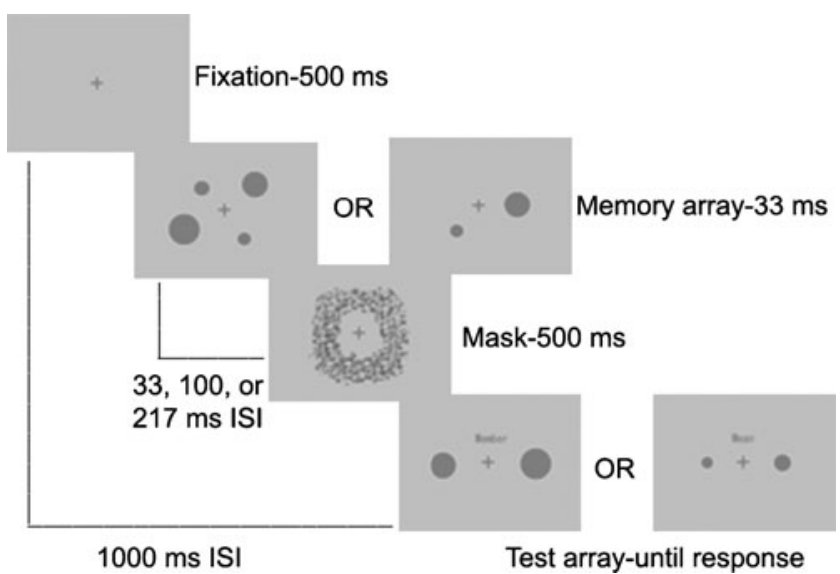

Fig. 1 Example of a trial in Experiment 1.

responded by pressing the appropriate keys from the keyboard. The participants were instructed to be as accurate as possible, without worrying about the speed of response. Since both tasks were based on sizes of disks that could not be articulated easily, we did not expect any interference from verbal working memory. The participants performed 4 trials per condition in the practice session and 23 trials per condition in the main experiment.

\section{Results and discussion}

The percentage accuracy data was submitted to a $2($ task : mean or member $) \times 2($ set - size : 2 or 4$) \times 3$ (SOA : 66, 133, or $250 \mathrm{~ms}$ ) repeated measures ANOVA. Overall, the participants were more accurate during the mean task $(70 \%)$ than during the member task $(61 \%), F(1,17)=$ 22.03, MSE $=175.13, p<.001$. The effect of SOA was significant, $F(2,34)=6.81, M S E=92.89, p<.01$. All post hoc comparisons were performed using Tukey's HSD test. Accuracy was better at the long SOA of $250 \mathrm{~ms}$ than at the shorter SOAs of $66 \mathrm{~ms}, t(17)=4.288, p<.05$, and $133 \mathrm{~ms}$, $t(17)=4.393, p<.05$. This is consistent with the general idea that working memory processes are capacity limited. Therefore, both the reduction in time for consolidation and the increase in memory load interfered with performance (Baijal et al., 2009; Jolicœur \& Dell'Acqua, 1998; Vogel et al., 2006).

The interaction between set size and task was significant, $F(1,17)=4.89, p<.05$ (see Fig. 2), which is consistent with findings from previous studies (Ariely, 2001; Baijal et al., 2009; Chong \& Treisman, 2005a). Accuracy was significantly better in the mean task than in member task for set size $4, t(17)=7.739, p<.001$. Performance in the member task was affected by set size, but performance in the mean task was not.

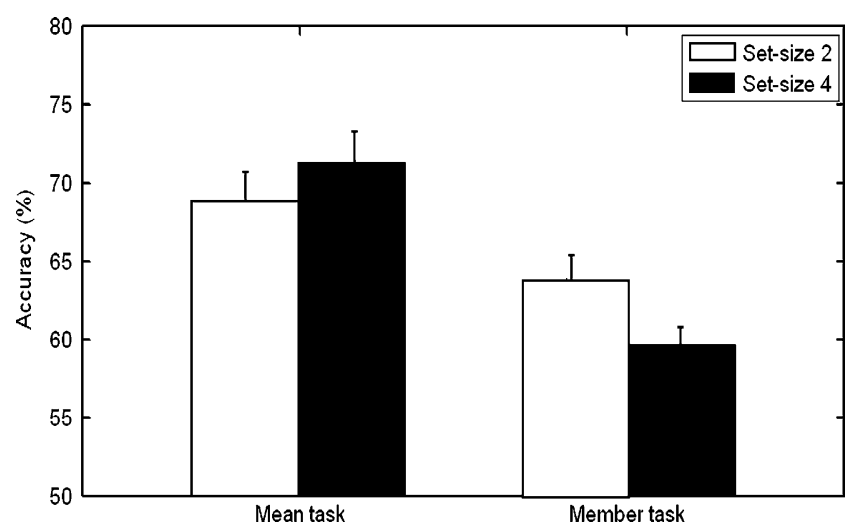

Fig. 2 Average accuracy in the mean and member tasks as a function of set size, showing better performance in the mean task, especially with set size 4 .

In addition, the interaction between task and SOA was significant, $F(2,34)=4.18, M S E=92.04, p<.05$ (see Fig. 3). Post hoc tests revealed that the accuracy was better in the mean task than in the member task at the SOA of $133 \mathrm{~ms}, t(17)=8.605, p<.001$. Also, the difference in accuracy between the mean and member tasks at $66 \mathrm{~ms}$ was close to significance, $t(17)=3.927, p=.11$. There was no difference in accuracy between the mean and member tasks at the long, 250-ms SOA. These results show that statistical information of a group of items is consolidated earlier than the information about the individual items themselves. Previous reports have indicated that working memory is influenced by interactions between the number of items, their complexity, and the exposure durations (Eng, Chen, \& Jiang, 2005). We redid the analysis with a 2 (task) $\times 3$

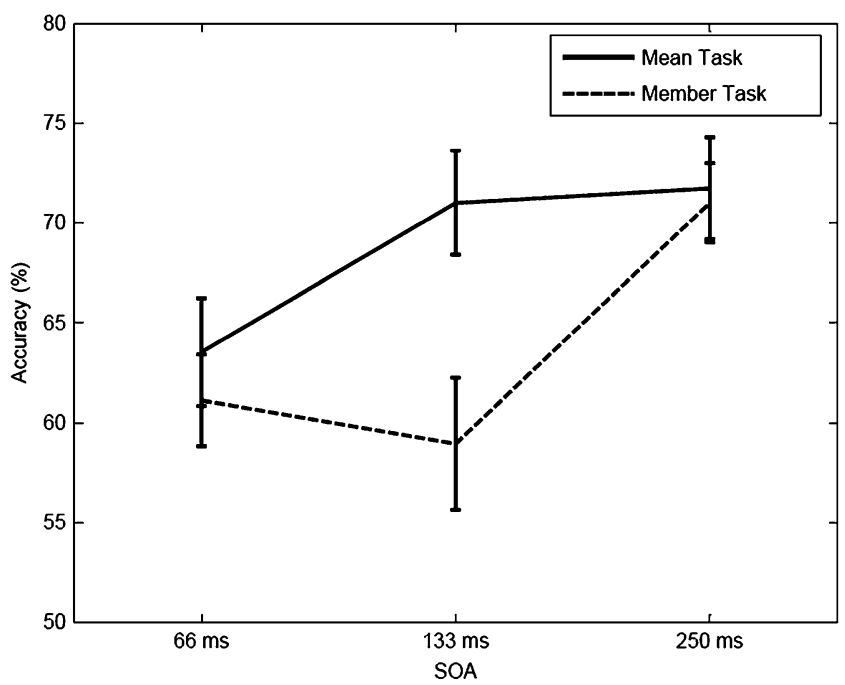

Fig. 3 Average accuracy in the mean and member tasks as a function of SOA. The difference in accuracy between the tasks emerges at the 133-ms SOA, when the accuracy is higher in the mean task than in the member task. 
(SOA) ANOVA on only set size 2 in order to check whether the interaction between task and SOA was present even when capacity limitations presumably did not play a critical role. Once again, the interaction between task and SOA was significant, $F(2,34)=5.299, M S E=63.72, p<.05$. Post hoc comparisons showed that the difference between the two tasks emerged at the intermediate SOA of $133 \mathrm{~ms}$, with better performance in the mean task than in the member task, $t(17)=6.419, p<.01$.

Overall, these results indicate that even when only two items have to be consolidated, less time (approximately half of the time) is needed to form the working memory representations of the statistical properties of items than when their individual identities have to be represented. In addition, this estimate is free from interference from a dual task or high working memory load. Given the natures of our tasks, it has been argued that they differ in terms of the scope of attention (Chong et al., 2008; Chong \& Treisman, 2003, 2005a, 2005b). In addition, there are differences in performance even with set size 2, indicating that these differences exist even for set sizes well within working memory capacity, indicating that the differences in consolidation are due to differences in the attentional processes involved in these tasks. However, it is possible that nonattentional factors might have affected working memory consolidation. Further studies would be needed to fully explore such a possibility. These results seem to demonstrate that working memory consolidation occurs at different rates during focused and distributed attention tasks, suggesting differences in capacity limitations between the tasks: Information in the distributed attention task was consolidated much earlier than information in the focused attention task. This is consistent with the view that statistical computations or the gist of a scene is obtained quickly (Chong \& Treisman, 2003; Treisman, 2006).

Previous studies (Chong \& Treisman, 2003, 2005a, 2005b) have indicated that the process of gist computation follows early perceptual grouping (such as by color) but precedes the limited-capacity bottleneck that forces focused attention. Specifically, the mean can be computed not just for different spatial areas - that is, for the left and right visual fields-but also for spatially intermingled sets containing targets and nontargets (Chong \& Treisman, 2005b) defined by color. Chong and Treisman explained this as early activation and inhibition from feature maps that could produce a candidate set of items to be averaged, without a need to bind several features. The results of our study also indicate that distributed attention mechanisms might be the default mode, as opposed to focused attention, which requires zooming in to bind features for object identification (Srivastava, Kumar, \& Srinivasan, 2010).

\section{Experiment 2}

An important related issue that needs to be addressed is whether the central mechanisms possibly associated with working memory consolidation are shared between the focused and distributed attention tasks. There is some evidence for the existence of interference when task resources must be shared between focused and distributed attention tasks (Chong \& Treisman, 2005b). The estimation of statistical properties is more compatible with a concurrent task in which attention is distributed over an entire display rather than focused on individual items. Similarly, tasks that have required individuating items have been more compatible with concurrent focused rather than distributed attention tasks. This indicates a competition between focused and distributed attention tasks for limited central resources. One possible way to investigate this issue is by using an additional task whose central processing requirements overlap with those of the primary task, resulting in interference between them (Jolicœur \& Dell'Acqua, 1998).

To investigate the dependence of focused and distributed attention tasks on common central processing resources, we decided to use a mixed block in which participants were not told in advance which task (mean or member) to perform in a given trial. The task to be performed was given to the participant after the stimulus had been presented, so that the same central processing resources would be used to perform the computations necessary to perform both tasks. We hypothesized that if common central processing resources were used by both of the tasks, performance in both of them would then decrease in the mixed block as compared to the single-task blocks. In addition, we specifically expected these effects to occur after the commencement of consolidation (i.e., at longer SOAs). A lack of effect in the mixed blocks could be taken as evidence for the use of independent pools of resources for these two tasks.

In Experiment 1, we used masks to disrupt the consolidation process at various time intervals in order to estimate the rate of consolidation for focused and distributed attention tasks. We found that with two items to be consolidated, the focused attention task required at least 200-250 ms for participants to form durable working memory representations (Vogel et al., 2006). However, for the distributed attention task, the time required for consolidation was much less, around 100-150 ms. Moreover, there was no difference in performance between the two tasks at the shortest SOA. Given these differences in consolidation, we compared performance in both of these tasks during single-task and mixed-task conditions using a longer SOA $(250 \mathrm{~ms})$, which would provide adequate time for consolidation and equivalent performance in the two tasks (as in Experiment 1). We also used the shortest SOA, $66 \mathrm{~ms}$, 
to test whether any effects could be due to the processes that occur before working memory consolidation.

\section{Method}

Participants A group of 18 volunteers from the University of Allahabad with normal or corrected-to-normal vision participated in the experiment.

Stimuli and apparatus The stimuli and apparatus were the same as in Experiment 1.

Procedure The procedure used in this experiment was identical to that used in Experiment 1, with the following exceptions. The SOA used was either 66 or $250 \mathrm{~ms}$. The single-task and mixed-task conditions were blocked (two blocks of trials for each of the single tasks, and four blocks for the mixed task), and the task type (mean or member single task, or mixed task) was indicated at the beginning of each block. The order of the single/mixed blocks was randomized across participants. During the single-task blocks, the participants performed either the member or the mean task. During the mixed-task block, the member and mean task trials were randomly intermixed within the block. If focused and distributed attention tasks use common central resources, the mixed-block task performance would then suffer. However, if focused and distributed attention tasks can be performed independent of each other, there would be no costs involved in the mixed-task scenario.

\section{Results and discussion}

Percentage accuracy data were submitted to a 2 (SOA : 133 or $250 \mathrm{~ms}) \times 2($ task : mean or member $) \times$ 2(type : single or mixed task) $\times 2$ (set size : 2 or 4 ) ANOVA. The results showed significant main effects of SOA, $F(1,17)=$ 4.28, $M S E=105.5, p=.05$, task, $F(1,17)=20.76, M S E=$ 183.7, $p<.001$, type, $F(1,17)=4.70, M S E=96.0, p<.05$, and set size, $F(1,17)=7.07, M S E=60.9, p<.05$. Overall, performance was better with the long SOA $(67 \%)$ than with the short SOA (64\%); for the mean task $(69 \%)$ than for the member task (62\%); for a single task $(67 \%)$ than for the mixed task (64\%); and with set size $2(67 \%)$ than with set size 4 (64\%). Further results showed set-size-dependent differences between the two tasks, indicated by a significant interaction effect between task and set size, $F(1,17)=5.67$, $M S E=249.9, p<.05$. The post hoc tests showed that with set size 4 , the accuracy was better in the mean task than in the member task, $t(17)=6.296, p<.01$. Moreover, in the member task, the accuracy for set size 2 was better than that for set size $4, t(17)=3.7, p=.07$, with no corresponding change in the mean task $(p>.4)$. These results were consistent with those of the Experiment 1.

The interaction effect between SOA and set size was also significant, $F(1,17)=26.56, M S E=24.2, p<.001$. The post hoc tests showed that the set-size-related differences (i.e., set size 2 was more accurate than set size 4 ) appeared only at the long SOA, $t(17)=9.371, p<.001$. The SOA-related differences (i.e., the long SOA elicited better accuracy than the short SOA) appeared only with set size $2, t(17)=9.474$, $p<.001$. This supports the general idea that working memory consolidation is a capacity-limited process (Jolicœur \& Dell'Acqua, 1998; Vogel et al., 2006). This is because the addition of more items resulted in an increase in the amount of time for the consolidation of visual representations. Conversely, with a reduction in the time for consolidation, fewer items could be remembered. Consistent with previous reports, the larger memory arrays required more uninterrupted time to allow for successful consolidation (Vogel et al., 2006).

There was a significant interaction between SOA, task, and type, $F(1,17)=4.512, M S E=65.3, p<.05$ (see Fig. 4). We were mainly interested in whether performance would be reduced in the mixed-task relative to the single-task condition for the member and mean tasks with respect to SOA. Therefore, to understand these effects better, we performed separate ANOVAs for the mean and member tasks, with SOA and Task Type as factors. The analysis with the mean task revealed a main effect of type, $F(1,17)=6.78$, $M S E=46.0, p<.05$, with accuracy in the single-task blocks being better than in the mixed-task blocks. In addition, the main effect of SOA was close to significance, $F(1,17)=3.05$, $M S E=64.9, p<.1$, with accuracy at the long SOA being marginally better than that at the short SOA.

The analysis of the member task revealed a significant interaction effect between SOA and type, $F(1,17)=6.74$, $M S E=44.9, p<.05$. Planned comparisons showed that there was a drop in the member task accuracy in the mixedtask condition at the long SOA, $t(17)=3.16, p<.05$, but not at the short SOA. In fact, the difference was in the opposite

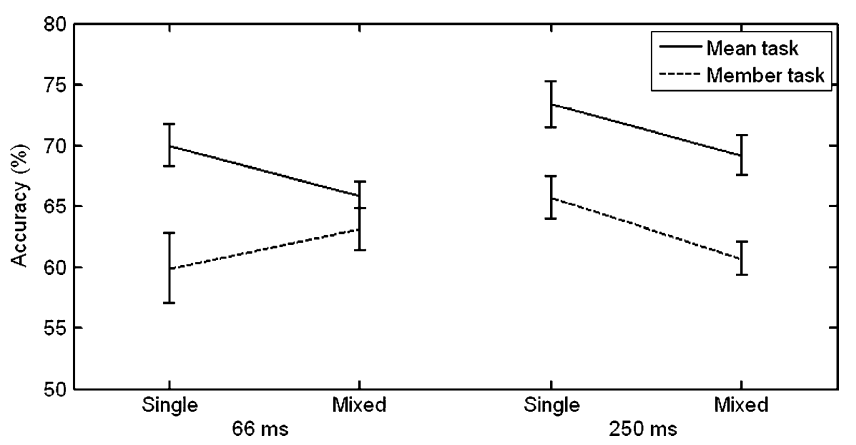

Fig. 4 Average accuracy in the mean task (solid line) and the member task (dashed line) of Experiment 2 as a function of block type and SOA 
direction, but the effect was not significant, $t(17)=2.02$, $p>$.1. Therefore, both mean and member tasks suffered during the mixed-task condition, suggesting interference of the tasks with each other for the capacity-limited central resources. In addition, the drop in accuracy in the member task was sensitive to SOA.

Chong and Treisman (2005b) provided evidence that the mean task interfered with a concurrent task if the latter required focused attention to individual items rather than global attention to the display as a whole. Consistent with this, the results of our study suggest that both the mean and member tasks are affected by sharing of central resources. In the visual search task used by Chong and Treisman (2005b), the study design did not control for the exposure duration of the stimuli. Therefore, the fact that the results of the mean task were compatible with the pop-out (global or distributed attention) rather than the conjunction search (focused attention) could be attributed to factors other than capacitylimited resources. In addition, in their other experiment, using the mean task with a concurrent task, the latter task required deployment of attention at different spatial scales. In our study, we matched the mean and member tasks on exposure duration and spatial scale specifically to test whether or not capacity-limited resources affect both tasks.

The task conditions used in Alvarez and Oliva (2008) were similar to those in the Experiment 2 of our study. For Alvarez and Oliva, the participants reported the location of either a single object or the centroid of multiple objects, with both conditions randomly intermixed. Although they found that the location of the centroid could be reported more accurately than that of an individual object, it may not be possible to test the utilization of capacity-limited central resources in this task due to multiple factors, such as long stimulus presentation times ( 6 or $10 \mathrm{~s}$ ) and the additional counting task performed by the participants. Additionally, the authors showed that this effect was not due to subsampling of one or two items. In this context, our results could be interpreted as reflecting interference between focused and distributed attention only when they compete for capacity-limited resources, not when sufficient resources are available.

Our finding indicates that both tasks tap into the same capacity-limited processes. However, the demands of sufficient time for consolidation may specifically impede the execution of the member task. On the other hand, the consolidation time requirements for the mean task are much less even when two distinct attention-demanding tasks have to be performed, because the allocation of central resources does not interact with the time of consolidation in the mean task. The lack of any differences in single- and mixed-task performance at the short SOA suggests that the sharing of central resources is very little, if at all, affected by processes occurring before working memory consolidation.

\section{General discussion}

Our study was motivated by recent studies arguing for differences in the working memory processes associated with the extraction of statistical information through distributed attention as compared to object identification using focused attention (Ariely, 2001; Baijal et al., 2009; Chong \& Treisman, 2003, 2005a, 2005b; Treisman, 2006). We tried to quantify the time course of consolidation in working memory during tasks that involved either focused or distributed attention by using masks to interrupt the encoding process at various time intervals and measured performance in the two types of tasks.

In general, using tasks that required working memory consolidation, we found that the participants were more efficient in performing the distributed than the focused attention task even when faced with larger memory arrays, which is consistent with previous reports (Ariely, 2001; Baijal et al., 2009; Chong et al., 2008; Chong \& Treisman, 2005a; Im \& Chong, 2009). This strengthens the claim that distributed attention is associated with relatively lower capacity limits than focused attention (Treisman, 2006). Most importantly, the study suggests that differences based on the type of attention exist in the efficiency of working memory consolidation and the use of central processing resources.

The results of Experiment 1 clearly indicate that the rate of consolidation varied depending on the type of attention deployed during the task. Statistical information (in the distributed attention task) was encoded about twice as fast as the detailed information (in the focused attention task) about objects. This difference in the time courses of consolidation in working memory has implications for several studies on the computation of statistical properties. For example, the processing advantage found in statistical property estimation tasks (Baijal et al., 2009; Chong \& Treisman, 2003, 2005a, 2005b) can be attributed to faster consolidation of information processed with distributed attention. In support of this finding, previous studies have indicated that statistical information is extracted from sufficiently early visual representations of objects (Im \& Chong, 2009). Therefore, the rate obtained with the distributed attention task might be the upper limit for consolidation time in working memory. Similar results have been obtained with other studies involving tasks that directly manipulated focused and diffused attention (Belopolsky, Zwaan, Theeuwes, \& Kramer, 2007). These showed that visual search was faster when attention was distributed than when it was focused, possibly due to parallel processing. In addition, an irrelevant singleton affected performance when attention was diffused rather than focused, indicating a wider window for distributed attention (Belopolsky et al., 2007). 
Another possible explanation is based on the "zoom lens" model of attention (Eriksen \& St. James, 1986). As indicated by the model, the scope of attention appears to be flexible, and the zooming in or out of attention depends on the task goals (Cowan et al., 2005). According to this adjustable-attention hypothesis, the scope of attention can zoom in to hold on to a goal (e.g., Kane et al., 2001) and zoom out to apprehend a maximal field of items (Chen, 2003; Usher et al., 2001). Other studies have shown differences in working memory storage capacity in which the only apparent storage requirement was to hold on to the goal of the task. These differences were obtained when interference with maintenance of the task goal had to be overcome by attentional control (Conway, Cowan, \& Bunting, 2001; Kane et al., 2001; Kane \& Engle, 2003).

In these views, attentional control is needed to actively maintain task-relevant representations in working memory. Based on the notion that domain-general attention control abilities are important for working memory capacity (Cowan et al., 2005), one possibility is that the additional time of consolidation observed in the focused attention task in our study could be associated with the time required for zooming in to the individual items. On the other hand, the broad scope of attention in the case of distributed attention (Baijal \& Srinivasan, 2009; Treisman, 2006) could be the default mode, in which attention is zoomed out. A recent study showed that distributed attention could be the default mode, enabling faster processing of global than of local information, as the latter requires attention to zoom in for identification of local components (Srivastava et al., 2010). This concept is also consistent with the attentional gradient model of LaBerge and Brown (1989), who proposed that there is a gradient of processing that becomes less intense as one gets away from the focus of attention. Therefore, the zoomed-out setting of distributed attention is responsible for obtaining coarse-level information, such as gist and statistical information.

In the second experiment, we found that the consolidation processes in focused and distributed attention tasks were affected when both tasks had to be executed. This shows that there was a joint demand for central resources for both of the tasks that resulted in interferences during the mixed-task condition. This demand for central resources was needed for consolidation in working memory, although not necessarily for extracting the visual information itself (Baijal et al., 2009). Thus, both modes of attention seem to rely on a common pool of central resources. Our study is also consistent with a previous report depicting interference when the mean task was performed with a concurrent task that involved focused attention (Chong \& Treisman, 2005a).

Our results indicate that consolidation in focused and distributed attention tasks may involve a unitary set of control mechanisms rather than a loose collection of independent processes. Yet, it is to be noted that the member task is particularly sensitive to the time requirements for the consolidation process. From a broader perspective, our results illustrate the levels of working memory representation of items in a visual scene-the summary representations akin to distributed attention are formed much earlier than the individualized object representations formed by focused attention.

Some of the differences in the consolidation requirements between focused and distributed attention tasks can be attributed to the resolution of working memory representations formed in these tasks. There is some evidence to show that attention biases capacity-limited resources to represent an increasing number of objects with decreasing precision (Bays \& Husain, 2008). When a large number of items are selected, the representations are coarse (Franconeri et al., 2007), as in the case of the distributed attention task. These coarse representations may require less time for consolidation. This is also indicated by research on memory representation for pictures: People can store more than four pictures in a short period of time (Nickerson, 1965), but the visual details of the pictures seem to be poorly retained (Intraub, 1997).

At first glance, the results from Experiment 1 of our study seem to support the claims of a flexible resource model, according to which mnemonic resources can be allocated in a continuous fashion, such that a smaller proportion of resources is left for each item (i.e., representations become coarser) as the number of items increases (see Fukuda, Awh, \& Vogel, 2010), as in the mean task. However, the results from Experiment 2 of our study show that a separate store of capacity-limited mnemonic resources exists that operates independent of the resolution (or coarseness) of visual representations, such that competition for these resources results in interference, even when coarse representations have to be formed (as in the mean task). More studies are needed to examine these aspects of working memory.

In conclusion, our study provides a working memory function-based view of the processes underlying focused and distributed attention. We demonstrated that the capacity limitations in terms of the time required for consolidation in working memory depend on the type of information required. However, the central processes that control working memory behavior are shared by focused and distributed attention. Exactly how brain regions cooperate to encode statistics versus detailed information requires further investigation.

\section{References}

Alvarez, G. A., \& Oliva, A. (2008). The representation of simple ensemble visual features outside the focus of attention. Psychological Science, 19, 392-398. 
Ariely, D. (2001). Seeing sets: Representation by statistical properties. Psychological Science, 12, 157-162.

Baddeley, A. (1997). Human memory: Theory and practice. Hove: Psychology Press.

Baijal, S., Nakatani, C., Srinivasan, N., \& van Leeuwen, C. (2009). Controlled access to working memory during statistical property judgment: An examination of distributed attention theory using event related potential measures (Prog. No. 381.15/FF131.2009). Neuroscience Meeting Planner. Chicago, IL: Society for Neuroscience.

Baijal, S., \& Srinivasan, N. (2009). Types of attention matter for awareness: A study with color afterimages. Consciousness and Cognition, 18, 1039-1048.

Bays, P. M., \& Husain, M. (2008). Dynamic shifts of limited working memory resources in human. Vision Science, 321, 851-854.

Belopolsky, A. V., Zwaan, L., Theeuwes, J., \& Kramer, A. F. (2007). The size of an attentional window modulates attentional capture by color singletons. Psychonomic Bulletin \& Review, 14, 934938.

Cavanagh, P., \& Alvarez, G. A. (2005). Tracking multiple targets with multifocal attention. Trends in Cognitive Sciences, 9, 349-354.

Chen, Z. (2003). Attentional focus, processing load, and Stroop interference. Perception \& Psychophysics, 65, 888-900. doi:10.3758/BF03194822

Chong, S. C., Joo, S. J., Emmanouil, T. A., \& Treisman, A. (2008). Statistical processing: Not so implausible after all. Perception \& Psychophysics, 70, 1327-1336.

Chong, S. C., \& Treisman, A. (2003). Representation of statistical properties. Vision Research, 43, 393-404.

Chong, S. C., \& Treisman, A. (2005a). Attentional spread in the statistical processing of visual displays. Perception \& Psychophysics, 67, 1-13.

Chong, S. C., \& Treisman, A. (2005b). Statistical processing: Computing the average size in perceptual groups. Vision Research, 45, 891-900.

Chun, M. M., \& Potter, M. C. (1995). A two-stage model for multiple target detection in rapid serial visual presentation. Journal of Experimental Psychology. Human Perception and Performance, $21,109-127$.

Conway, A. R. A., Cowan, N., \& Bunting, M. F. (2001). The cocktail party phenomenon revisited: The importance of working memory capacity. Psychonomic Bulletin \& Review, 8, 331-335. doi:10.3758/BF03196169

Cowan, N. (1998). Visual and auditory working memory capacity. Trends in Cognitive Sciences, 2, 77-78.

Cowan, N. (2001). The magical number 4 in short-term memory: A reconsideration of mental storage capacity. The Behavioral and Brain Sciences, 24, 87-185.

Cowan, N., Elliott, E. M., Saults, J. S., Morey, C. C., Mattox, S., Hismjatullina, A., et al. (2005). On the capacity of attention: Its estimation and its role in working memory and cognitive aptitudes. Cognitive Psychology, 51, 42-100.

Eng, H. Y., Chen, D., \& Jiang, Y. (2005). Visual working memory for simple and complex visual stimuli. Psychonomic Bulletin \& Review, 12, 1127-1133.

Eriksen, C. W., \& St. James, J. D. (1986). Visual attention within and around the field of focal attention: A zoom lens model. Perception \& Psychophysics, 40, 225-240.

Franconeri, S. L., Alvarez, G. A., \& Enns, J. T. (2007). How many locations can be selected at once? Journal of Experimental Psychology. Human Perception and Performance, 33, 1003-1012.
Fukuda, K., Awh, E., \& Vogel, E. K. (2010). Discrete capacity limits in visual working memory. Current Opinion in Neurobiology, 20 , 177-182.

Im, H. Y., \& Chong, S. C. (2009). Computation of mean size is based on perceived size. Attention, Perception, \& Psychophysics, 71, 375-384.

Intraub, H. (1997). The representation of visual scenes. Trends in Cognitive Sciences, 1, 217-222.

Jolicœur, P., \& Dell'Acqua, R. (1998). The demonstration of shortterm consolidation. Cognitive Psychology, 36, 138-202.

Kane, M. J., Bleckley, M. K., Conway, A. R. A., \& Engle, R. W. (2001). A controlled-attention view of working-memory capacity. Journal of Experimental Psychology. General, 130, 169-183. doi:10.1037/0096-3445.130.2.169

Kane, M. J., \& Engle, R. W. (2003). Working-memory capacity and the control of attention: The contributions of goal neglect, response competition, and task set to Stroop interference. Journal of Experimental Psychology. General, 132, 47-70. doi:10.1037/ 0096-3445.132.1.47

LaBerge, D., \& Brown, V. (1989). Theory of attentional operations in shape identification. Psychological Review, 96, 101-124.

Luck, S. J., \& Vogel, E. K. (1997). The capacity of visual working memory for features and conjunctions. Nature, 390, 279-281.

Makovski, T., \& Jiang, Y. V. (2007). Distributing versus focusing attention in visual short-term memory. Psychonomic Bulletin \& Review, 14, 1-7.

Nickerson, R. S. (1965). Short-term memory for complex meaningful visual configurations: A demonstration of capacity. Canadian Journal of Psychology, 19, 155-160.

Potter, M. C. (1976). Short-term conceptual memory for pictures. Journal of Experimental Psychology: Human Learning and Memory, 2, 509-522.

Srinivasan, N., Srivastava, P., Lohani, M., \& Baijal, S. (2009). Focused and distributed attention. Progress in Brain Research, 176, 87-100.

Srivastava, P., Kumar, D., \& Srinivasan, N. (2010). Time course of visual attention across perceptual levels and objects. Acta Psychologica, 135, 335-342.

Treisman, A. (1988). Features and objects: The Fourteenth Bartlett Memorial Lecture. The Quarterly Journal of Experimental Psychology, 40, 201-237.

Treisman, A. (2006). How the deployment of attention determines what we see. Visual Cognition, 14, 411-443.

Usher, M., Cohen, J. D., Haarmann, H., \& Horn, D. (2001). Neural mechanism for the magical number 4: Competitive interactions and nonlinear oscillation. The Behavioral and Brain Sciences, 24, $151-152$.

Vogel, E. K., Luck, S. J., \& Shapiro, K. L. (1998). Electrophysiological evidence for a postperceptual locus of suppression during the attentional blink. Journal of Experimental Psychology. Human Perception and Performance, 24, 1656-1674.

Vogel, E. K., Woodman, G. F., \& Luck, S. J. (2006). The time course of consolidation in visual working memory. Journal of Experimental Psychology. Human Perception and Performance, 6, 1436-1451.

Ward, R., Duncan, J., \& Shapiro, G. (1996). The slow time-course of visual attention. Cognitive Psychology, 30, 79-109.

Wolfe, J. M. (1994). Guided Search 2.0: A revised model of visual search. Psychonomic Bulletin \& Review, 1, 202-238.

Woodman, G. F., \& Luck, S. J. (1999). Electrophysiological measurement of rapid shifts of attention during visual search. Nature, 400, 867-869. 